\title{
Study on Electrochemical Processes of NiO Model Electrode during the First Lithiation/delithiation Cycle via Electrochemical Impedance Spectroscopy
}

\author{
Lei Wang, Haixia $\mathrm{Wu}^{*}$ and Shouwu Guo* \\ Department of Electronic Engineering, School of Electronic Information and Electrical Engineering, \\ Shanghai Jiao Tong University, Shanghai 200240, P. R. China \\ *E-mail: haixiawu@ @jtu.edu.cn, swguo@sjtu.edu.cn
}

doi: $10.20964 / 2018.12 .51$

Received: 9 July 2018 / Accepted: 10 August 2018 / Published: 5 November 2018

\begin{abstract}
Transition metal oxides (TMOs) have been researched as active electrode materials for Li-ion batteries for over 20 years owing to their large specific capacity based on conversion reactions rather than intercalation/deintercalation mechanism. Here, $\mathrm{NiO}$ was chosen not only as a representative among those TMOs but also as a model electrode material to fundamentally study its electrochemical processes during the first lithiation/delithiation cycle via electrochemical impedance spectroscopy (EIS). The NiO model electrode was constructed by electrodepositing a pure $\mathrm{NiO}$ film on the surface of the copper foil and directly served as a working electrode in Li-ion half cells without any other additives. These cells were stopped at certain states in the first lithiation/delithiation cycle and ready for the EIS test. Interestingly, other two semicircles were found in Nyquist plots, which were ascribed to $\mathrm{NiO}$ and structure transformation besides the normal two semicircles related to solid electrolyte interphase (SEI) and the interface between $\mathrm{NiO}$ and electrolyte. Furthermore, bounded diffusion was more suitable than semi-infinite diffusion to explain $\mathrm{Li}^{+}$diffusion process in conversion-type electrodes. Thus, a new fitting circuit was proposed and the electrochemical processes of the NiO model electrode were studied quantitatively according to fitted data.
\end{abstract}

Keywords: NiO, EIS, Li-ion battery, fitting circuit

\section{$\underline{\text { FULL TEXT }}$}

(C) 2018 The Authors. Published by ESG (www.electrochemsci.org). This article is an open access article distributed under the terms and conditions of the Creative Commons Attribution license (http://creativecommons.org/licenses/by/4.0/). 